\title{
HOTAIR contributes to the growth of liver cancer via targeting $\mathrm{miR}-217$
}

\author{
LI-PING WANG $^{1}$, JUN-PING WANG ${ }^{2}$ and XIN-PING WANG ${ }^{3}$ \\ ${ }^{1}$ Department of Medicine, Xi'an Honghui Hospital, Xi'an, Shaanxi 710068; ${ }^{2}$ Department of Medicine, \\ The Friendship Hospital of Shaanxi, Xi'an, Shaanxi 710000; ${ }^{3}$ Department of General Surgery, \\ Xi'an No. 4 Hospital, Xi'an, Shaanxi 710004, P.R. China
}

Received March 28, 2017; Accepted February 23, 2018

DOI: $10.3892 / \mathrm{ol} .2018 .8341$

\begin{abstract}
Non-coding RNAs are important in the progression of liver cancer. The present study aimed to investigate the effects of long non-coding RNA HOX transcript antisense RNA (HOTAIR) on the proliferation of liver cancer and the association between HOTAIR and microRNA (miR)-217. It was demonstrated that the expression of HOTAIR was upregulated in liver cancer tissues and 3 liver cancer cell lines (MHCC97H, HepG2 and Hep3B). Inhibition of HOTAIR with HOTAIR small interfering (si) RNA lentiviral vectors significantly suppressed the cell proliferation of HepG2 cells, and downregulated the protein expression levels of two proliferation markers, Ki67 and proliferating cell nuclear antigen (PCNA). Furthermore, inhibition of HOTAIR induced G0/G1 cycle arrest by increasing the expression of p27 and decreasing the expression of cyclin D1. It was then predicted and verified that miR-217 was the target of HOTAIR. Expression of miR-217 was downregulated in liver cancer tissues and the 3 liver cancer cell lines. Further results revealed that inhibition of HOTAIR markedly upregulated the expression of miR-217 in HepG2 cells, and miR-217 inhibitor-induced reduction of miR-217 was significantly suppressed by HOTAIR inhibition. Furthermore, the increased cell proliferation and growth, the upregulated expression of Ki67 and PCNA, and the reduced G0/G1 cycle arrest induced by miR-217 inhibitor were partly rescued by inhibition of HOTAIR. Finally, the in vivo experiment indicated that HOTAIR inhibition suppressed tumorigenesis, including the smaller tumor volume and the
\end{abstract}

Correspondence to: Dr Xin-Ping Wang, Department of General Surgery, Xi'an No. 4 Hospital, 21 Jiefang Road, Xincheng, Xi'an, Shaanxi 710004, P.R. China

E-mail: xinpingwangxpw@126.com

Abbreviations: HOTAIR, HOX transcript antisense RNA; miR-217, microRNA-217; PCNA, proliferating cell nuclear antigen; ESCC, esophageal squamous cell carcinoma; 3'-UTRs, 3'-untranslated regions

Key words: long non-coding RNA, liver cancer, cell proliferation, cycle arrest, microRNA-217 reduced levels of Ki67. Overall, HOTAIR contributes to the proliferation and growth of liver cancer via downregulation of miR-217.

\section{Introduction}

Liver cancer is one of the most malignant tumors with increasing incidence and cancer-related mortality worldwide (1). In recent years, great advances in the treatment for liver cancer have been achieved, including surgical technique, radiotherapy and chemotherapy. However, the long-term survival rate is still low among liver cancer patients due to the aggressive metastasis and recurrence. Currently, a variety of studies have revealed that the progression of liver cancer was closely associated with multiple abnormal molecular expression and dysfunctional signaling pathways, such as tumor protein P53, Wnt/ $\beta$-catenin signaling, NF- $\kappa \mathrm{B}$ signaling, and non-coding RNAs (2-5). However, the precise molecular mechanisms underlying liver cancer progression still urgently need to be established, which may contribute to improve therapeutic strategies for liver cancer patients.

Long non-coding RNAs (lncRNAs) are non-protein coding RNAs longer than 200 nucleotides. Over the recent years, studies has shown that lncRNAs have significant functions in a variety of biological processes, including cell proliferation, apoptosis, metastasis and inflammation $(6,7)$. It has been reported that aberrant expression of lncRNAs is involved in the progression of numerous cancer diseases. Long non-coding RNA MALAT1 could interact with miR-124 to modulate tongue cancer growth (8), and MALAT1 also could promote the tumorigenicity and metastasis of gastric cancer (9). Upregulation of long non-coding RNA PlncRNA-1 promotes proliferation and induces epithelial-mesenchymal transition in prostate cancer (10), and XIST promotes the proliferation of pancreatic cancer by regulating miR-133a/EGFR (11). These investigations suggest that targeting lncRNAs may be a new strategy for diagnosis and therapy in cancers. The lncRNA HOX transcript antisense RNA (HOTAIR) is expressed form the Homeobox C (HOXC) locus on chromosome 12, which has been identified to be highly expressed and involved in poor prognosis in several cancers, such as breast cancer (12), colorectal cancer (13), pancreatic cancer (14), non-small cell lung cancer (15), and esophageal squamous cell carcinoma 
(ESCC) (16). Recently, several studies have revealed that HOTAIR has an abnormal expression in liver cancer and is closely associated with the progression and recurrence of liver cancer $(5,17,18)$. However, the role and underlying molecular mechanism of HOTAIR in liver cancer is still required further investigation.

MicroRNAs (miRNAs) are 20-25 nt non-coding RNAs that regulate the expression of genes by binding to the 3'-untranslated regions (UTRs) of target mRNAs, and thus, the regulation of miRNAs in many biological processes including proliferation, cycle arrest, and apoptosis is of great significance $(19,20)$. Recently, emerging evidence has demonstrated that the aberrant expression of miRNAs was involved in the progression of many cancers, including liver cancer. Several investigations have reported the regulationship of IncRNAs and miRNAs in controlling liver cancer progression. One recent study has shown that the oncogenic function of HOTAIR in liver cancer is partly based on the negative regulation of miR-1 (21). Additionally, miR-217 has been shown to function as a potential tumor suppressor in liver cancer progression (22). However, whether HOTAIR could promote the growth of liver cancer by regulating miR-217 is still not clear.

In the present study, we investigated the effects of HOTAIR on the proliferation and cycle arrest in liver cancer cell lines and specimens, and explored the regulation of HOTAIR on miR-217.

\section{Materials and methods}

Liver cancer tissues. Twenty-five paired liver cancer tissues and adjacent non-cancerous liver tissues were obtained from patients who underwent partial liver resection at Xi'an Honghui Hospital. Informed consent was obtained from all the patients. The study was performed in accordance with the Helsinki Declaration and was approved by the Human Ethics Committee/Institutional Review Board of Xi'an Honghui Hospital.

Cell culture. The normal human hepatic cell line HL-7702 and liver cancer cell lines (MHCC 97H, HepG2 and Hep3B) were obtained from American Type Culture Collection (Manassas, VA, USA). The HepG2 cell line used in this study was reported as misidentified, this cell line was originally thought to be a hepatocellular carcinoma cell line but was later shown to derive from a hepatoblastoma (23). All the cells were maintained in Dulbecco's modified Eagle's medium (DMEM) supplemented with $10 \%$ fetal bovine serum (FBS) and $1 \%$ penicillin/streptomycin, and cultured in a humidified cell incubator with an atmosphere of $5 \% \mathrm{CO}_{2}$ at $37^{\circ} \mathrm{C}$.

Construct and infection of HOTAIR siRNA lentiviral vector. The oligonucleotides encoding siRNA that directed against HOTAIR were synthesized by GenePharma Co., Ltd. (Shanghai, China) and annealed into double strands. Then the siRNA was inserted into the LV10-CMVRFP-Puro vector (GenePharma) and the constructed plasmids were confirmed by DNA sequencing. The plasmid DNAs, along with packaging vectors, were transiently transfected into HEK293T cells using Lipofectamine 2000 (Invitrogen) according to the manufacturer's instructions. The virus particles were collected $72 \mathrm{~h}$ after transfection and purified by ultracentrifugation. For transfection, HepG2 cells were seeded in the cell plates for $24 \mathrm{~h}$, and then infected with the HOTAIR siRNA lentiviral vectors or empty lentiviral vectors for control. qRT-PCR was performed to detect the infection efficiency after $72 \mathrm{~h}$.

RNA extraction, northern blotting and qRT-PCR. Total RNA from frozen liver cancer and paired non-cancerous liver tissues or cell lines were extracted with TRIzol reagent (Invitrogen; Thermo Fisher Scientific, Inc., Waltham, MA, USA) according to the manufacturer's instructions. For northern blot analysis, RNA samples were resolved in the agarose gel and then were transferred to nylon membrane, and hybridized with HOTAIR specific biotin-labeled DNA probes. HRP conjugated secondary antibodies were used and visualized with a ChemiDoc XRS imaging system. For qRT-PCR, cDNA was synthesized by reverse transcribing using a PrimeScript RT reagent kit (Takara, Dalian, China). The expression levels of HOTAIR and miR-217 were detected using the SYBR Premix Ex Taq kit (Takara). The relative expression levels were calculated using the $2^{-\Delta \Delta C t}$ method. $\beta$-actin was used as an internal control for HOTAIR, and U6 snRNA was used for miR-217.

CCK- 8 assay. Cell proliferation was measured using CCK-8 assay according to the manufacturer's protocol. Briefly, HepG2 cells were seeded into 96-well plates and treated according to different experimental requirements. Each well were incubated with $10 \mu \mathrm{l}$ CCK- 8 solution at different time points $(0,24$, 48,72 and $96 \mathrm{~h}$ ) for $4 \mathrm{~h}$ at $37^{\circ} \mathrm{C}$. The absorbance was measured at $450 \mathrm{~nm}$ using a spectrophotometer.

Western blotting. Total protein was extracted from liver cancer tissues and different treated HepG2 cells by using RIPA protein extraction reagent supplemented with $1 \mathrm{mM}$ phenylmethanesulfonyl fluoride (PMSF). The concentration of protein was measured by BCA protein assay (Tiangen, Beijing, China). Then $20 \mu \mathrm{g}$ of protein was separated by SDS-PAGE and transferred onto a PVDF membrane (EMD Millipore, Billerica, MA, USA). The membranes were blocked in 5\% non-fat milk for $1 \mathrm{~h}$ at room temperature, and then were probed with the primary antibodies overnight at $4^{\circ} \mathrm{C}$ : Anti-Ki67, anti-proliferating cell nuclear antigen (PCNA), anti-p27, anti-cyclin D1 and anti-GAPDH (Abcam, Cambridge, UK). Then the membranes were incubated with the corresponding HRP-conjugated secondary antibodies for $1 \mathrm{~h}$ at room temperature, followed by detection and visualization using a ChemiDoc XRS imaging system and analysis software (Bio-Rad Laboratories, Inc., Hercules, CA, USA).

Flow cytometry. HepG2 cells were seeded on 6-well plates for $24 \mathrm{~h}$, followed by treatment according to different experimental requirements. The cells were harvested and washed with PBS twice and suspended in cold phosphate-buffered saline (PBS). Cells were then stained with PI/RNase staining solution in dark for $30 \mathrm{~min}$ at $37^{\circ} \mathrm{C}$, and analyzed using flow cytometry.

Cell transfection. The miR-217 mimic or inhibitor and corresponding negative control were purchased from GenePharma. Cells transfection was carried out using Lipofectamine 2000 according to the manufacturer's instructions. Cells were harvested $48 \mathrm{~h}$ after transfection for further experiments. 


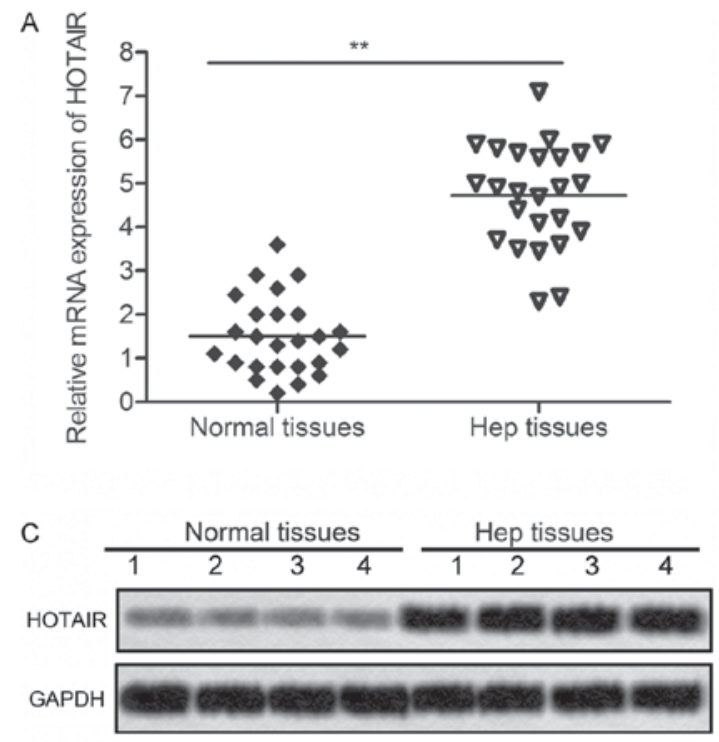

B

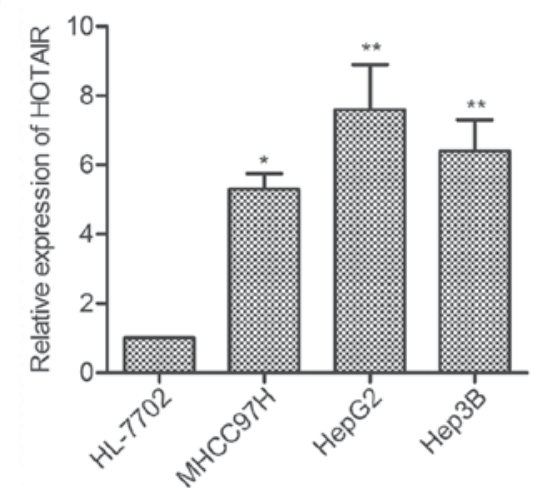

D

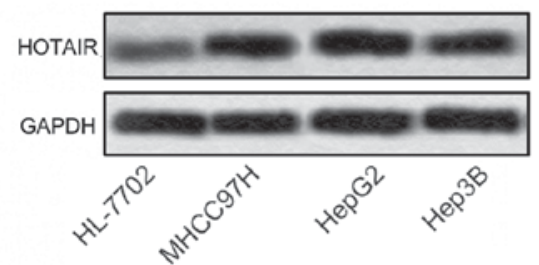

Figure 1. Expression of HOTAIR was upregulated in liver cancer tissues and liver cancer cell lines. (A) The expression of HOTAIR in tumor tissues and normal tissues was detected by qRT-RCR. (B) The expression of HOTAIR in 3 liver cancer cell lines (MHCC 97H, HepG2 and Hep3B) and normal human hepatic cell line HL-7702 was evaluated by qRT-RCR. (C) The expression of HOTAIR in tumor tissues and normal tissues was measured by northern blotting. (D) The expression of HOTAIR in MHCC 97H, HepG2, Hep3B and HL-7702 cell lines was measured by northern blotting ("P<0.05, $\left.{ }^{* *} \mathrm{P}<0.01\right)$. HOTAIR, HOX transcript antisense RNA

Dual luciferase reporter gene assays. The fragment from HOTAIR containing the predicted miR-217 binding site was amplified by PCR and cloned into a pGL3 luciferase reporter vector and this vector was named HATAIR WT. To test the binding specificity, the corresponding mutant was created by mutating the miR-217 seed region binding site and this vector was named HOTAIR MUT. HepG2 cells were seeded in 96-well plates, and after $24 \mathrm{~h}$, the cells were co-transfected with miR-217 mimics and pGL3 vectors containing HOTAIR WT or HOTAIR MUT. The luciferase activities was measured using Dual Luciferase reporter assay system (Promega Corporation, Madison, WI, USA) 48 h after transfection according to the manufacturer's instructions.

Xenograft transplantation in vivo. Four-week-old BALB/c male nude mice were purchased from The Experimental Animal Center of Xi'an Jiaotong University and maintained under specific pathogen-free conditions. The HepG2 cells stably transfected with HOTAIR siRNA lentiviral vector or empty lentiviral vector were injected into nude mice. Cell suspension $(100 \mu \mathrm{l})$ containing $1 \times 10^{7}$ cells was injected subcutaneously into the back of each nude mouse. The tumor size was measured every 6 days and on the 30th day following injection, all mice were sacrificed to recover the tumors. A portion of each tumor was fixed in $4 \%$ paraformaldehyde and embedded in paraffin for immunohistochemistry assay, and another portion was used for qRT-PCR and northern blotting. All the animal experiments were performed according to relevant national and international guidelines and were approved by the Animal Experimental Ethical Committee.

Immunohistochemistry assay. Tumor tissues were fixed with $4 \%$ paraformaldehyde, dehydrated, embedded in paraffin and cut into $4 \mu \mathrm{m}$ sections. The specimens were deparaffinized in xylene and rehydrated by using a series of graded alcohols. The tissue sections were immunohistochemically stained using the primary antibody anti-Ki67 overnight at $4^{\circ} \mathrm{C}$, and then incubated with horseradish peroxidase-conjugated secondary antibody $1 \mathrm{~h}$ at room temperature. Then the sections were incubated with the Cell and Tissue Staining Kit HRP-DAB system (R\&D Systems, MN, USA) according to the manufacturer's instructions.

Statistical analysis. Statistical analysis was carried out with GraphPad Prism 5.0 (GraphPad Software, Inc., La Jolla, CA, USA). The statistical significance between 2 groups was evaluated using Student's t-test. Each value was presented as mean \pm standard error of the mean (SEM) of at least three independent experiments. $\mathrm{P}<0.05$ was considered statistically significant.

\section{Results}

Expression of HOTAIR was upregulated in liver cancer tissues and liver cancer cell lines. To investigate the effects of HOTAIR in liver cancer, expression of HOTAIR was detected in 25 liver cancer tissues and 3 liver cancer cell lines by qRT-PCR and northern blotting, respectively. As shown in Fig. 1A and C, the expression of HOTAIR was significantly upregulated in the liver cancer tissues as compared with the normal tissues. Consistent with the result, the expression of HOTAIR were markedly increased in 3 liver cancer cell lines (MHCC97H, HepG2 and Hep3B) compared with normal human hepatic cell line HL-7702 (Fig. 1B and D). These results suggest that HOTAIR may be involved in the progression of liver cancer. 
A

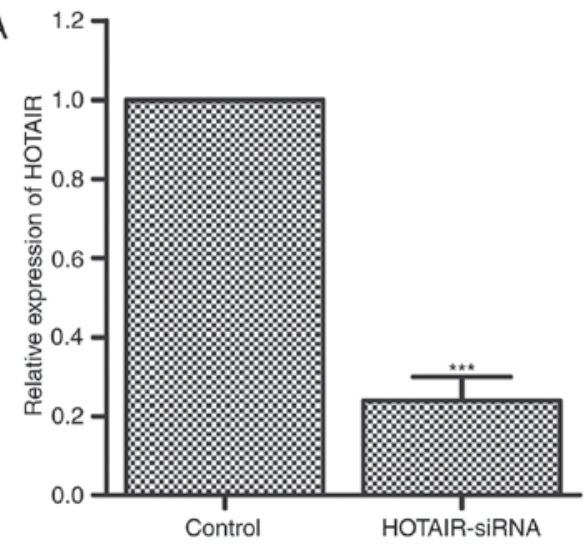

C

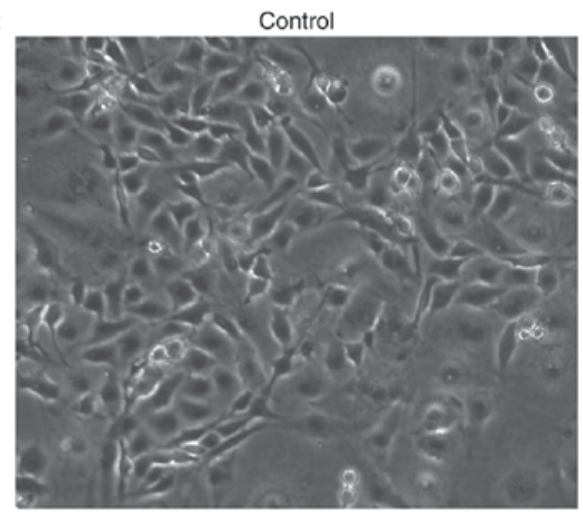

D

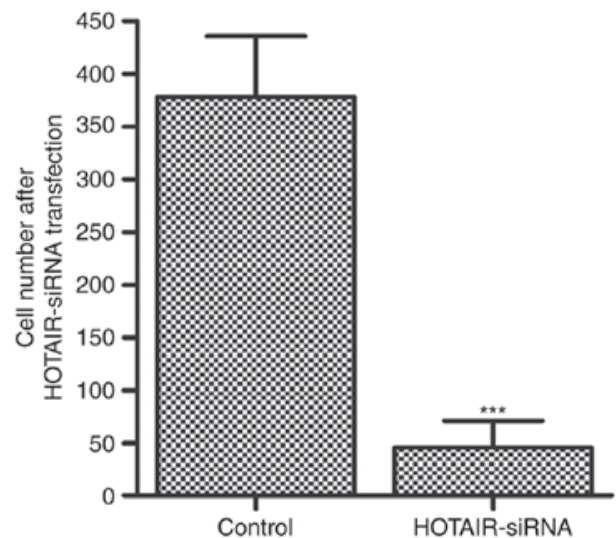

B
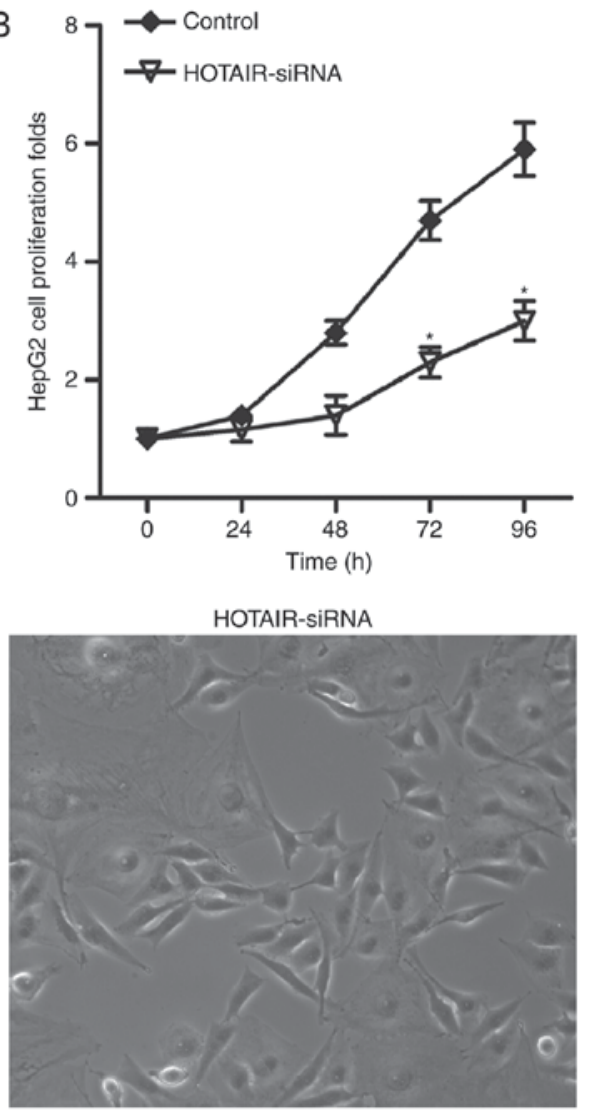

E
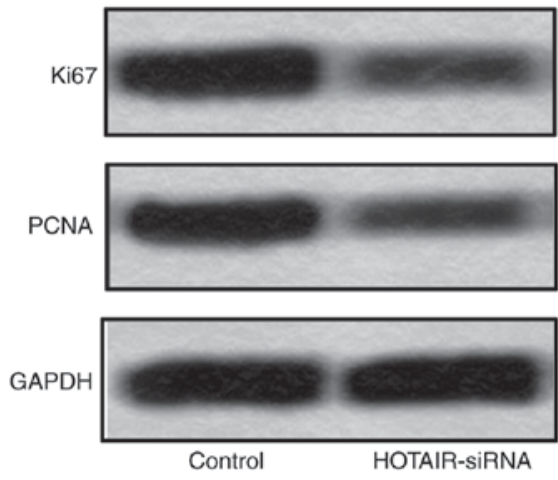

Figure 2. HOTAIR inhibition suppressed the proliferation of HepG2 cells. HepG2 cells were infected with HOTAIR siRNA lentiviral vectors or the empty lentiviral vectors for $72 \mathrm{~h}$. (A) The expression of HOTAIR was evaluated by qRT-RCR. (B) The proliferation rate was measured by CCK-8 assay. (C and D) The cell number was assessed by optical microscope. (E) The protein expression of two proliferation marker proteins Ki67 and PCNA was evaluated by western blotting. GAPDH was used as an internal control ( $\left.\mathrm{P}<0.05,{ }^{* * *} \mathrm{P}<0.001\right)$. HOTAIR, HOX transcript antisense RNA.

HOTAIR inhibition suppressed the proliferation of HepG2 cells. To investigate the effects of HOTAIR on the proliferation in liver cancer cells, the expression of HOTAIR was interfered by transfecting HOTAIR siRNA lentiviral vectors into HepG2 cells $(24,25)$. The interference efficiency was measured by RT-PCR after $72 \mathrm{~h}$, and the result was shown in Fig. 2A. The expression level of HOTAIR was significantly downregulated by $80 \%$ in HOTAIR-siRNA group as compared with the control group. The cell proliferation was measured by CCK- 8 assay at $24,48,72$ and $96 \mathrm{~h}$. The results showed that cell proliferation in HOTAIR-siRNA group had an obvious suppression at 72 and $96 \mathrm{~h}$ compared with control group (Fig. 2B). These results suggest that inhibition of HOTAIR plays an important role in the proliferation suppression in HepG2 cells. The results were confirmed by cell counting using optical microscope. As shown in Fig. 2C and D, the cell number had an obvious reduction after HOTAIR-siRNA lentiviral vectors infection. Furthermore, we detected the protein expression levels of two proliferation-associated markers, Ki67 and PCAN, by western blotting. As shown in Fig. 2E, the expression levels of Ki67 and PCAN were significantly inhibited in HOTAIR-siRNA group as compared with the control group. These results indicate that inhibition of HOTAIR suppresses the proliferation of HepG2 cells.

HOTAIR inhibition induced G0/G1 cell cycle arrest. Cell cycle arrest was measured by flow cytometry to further convince 
A
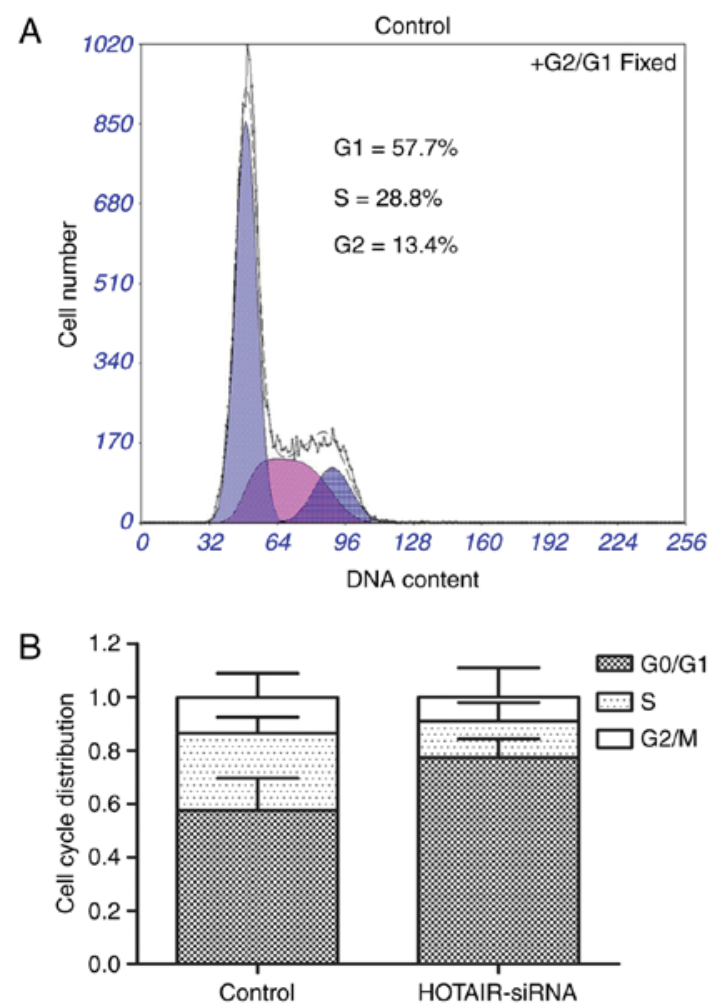
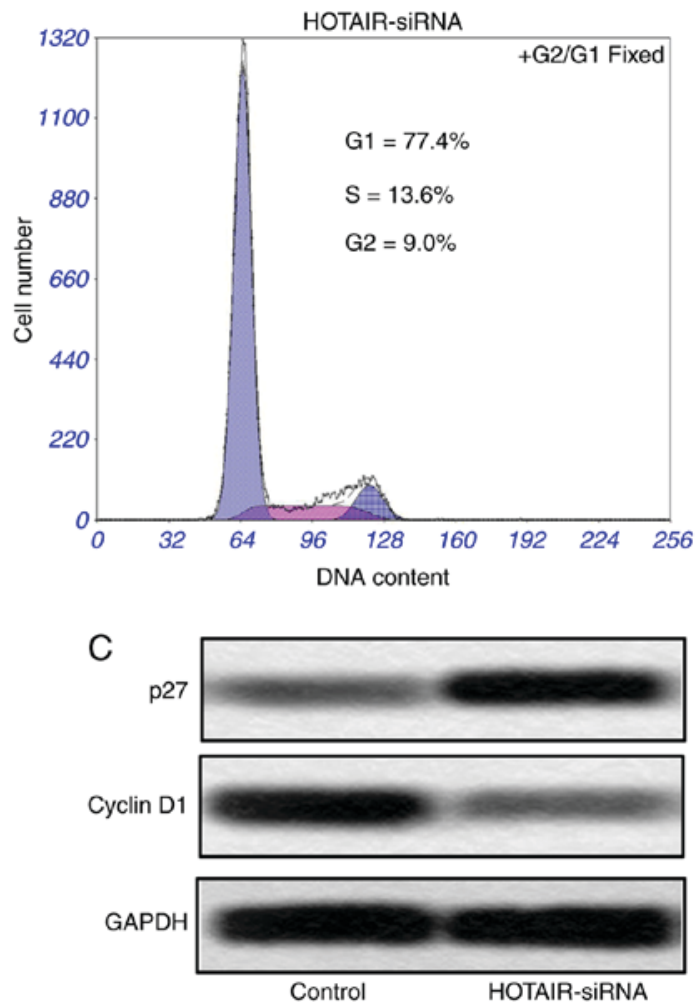

Figure 3. HOTAIR inhibition induced G0/G1 cell cycle arrest. (A and B) The cell cycle arrest was evaluated by flow cytometry. (C) The protein expression of two cell cycle markers p27 and cyclin D1 was evaluated by western blotting. GAPDH was used as an internal control. HOTAIR, HOX transcript antisense RNA.

the suppressing effect of HOTAIR siRNA on the proliferation in HepG2 cells. As shown in Fig. 3A and B, the G0/G1 cell cycle arrest was significantly increased in HOTAIR-siRNA group compared with control group. Moreover, we measured the protein expression levels of two cell cycle markers p27 and cyclin D1, and the results showed that the expression of p27 had an obvious upregulation, while the level of cyclin D1 had a significant downregulation in HOTAIR-siRNA group as compared with control group (Fig. 3C). These results suggest that inhibition of HOTAIR induces G0/G1 cell cycle arrest in HepG2 cells.

Expression of miR-217 was downregulated in liver cancer tissues and liver cancer cell lines. The targeting relationship between HOTAIR and miR-217 was predicted by StarBase v2.0 (http://starbase.sysu.edu.cn/) (Fig. 4A). We then detected the expression of miR-217 in 25 liver cancer tissues and 3 liver cancer cell lines by RT-PCR and northern blotting, respectively. As shown in Fig. $4 \mathrm{~B}$ and $\mathrm{D}$, the expression of miR-217 had a significantly downregulation in the liver cancer tissues as compared with the normal tissues. Consistent with the result, Expression of miR-217 were markedly downregulated in 3 liver cancer cell lines compared with normal human hepatic cell line HL-7702 (Fig. 4C and E). These results suggest that miR-217 plays a crucial role in the progression of liver cancer, and the expression between HOTAIR and miR-217 has a close correlation.

miR-217 is a direct target of HOTAIR. To further confirm the regulationship of HOTAIR and miR-217, we co-transfected
HepG2 cells with HOTAIR siRNA lentiviral vectors and miR-217 mimics or inhibitors. As shown in Fig. 5A, compared with miR-217 mock group, the expression of miR-217 in HepG2 cells was significantly downregulated when co-transfected with HOTAIR lentvirus vectors and miR-217 mock. The elevated level of miR-217 was also decreased adding HOTAIR lentvirus vectors in HepG2 cells transfected with miR-217 mimic. As shown in Fig. 5B, when HepG2 cells were co-transfected with HOTAIR siRNA lentvirus vectors and miR-217 inhibitors, the inhibitor-induced suppression of miR-217 was significantly reduced, and the expression of miR-217 was markedly increased as compared with that in cells transfected with miR-217 inhibitors alone. These results imply that HOTAIR regulate the expression of miR-217 in HepG 2 cells. In addition, we performed the Dual luciferase reporter assay to identify the targeting relationship between HOTAIR and miR-217, the results showed that miR-217 mimics transfection markedly decreased the relative luciferase activity of the plasmid containing HOTAIR sequences (WT) as compared with that containing mutating sequences (Fig. 5C). We further found that there was a negative regulation between the expression of HOTAIR and miR-217 in liver cancer tissues (Fig. 5D). Taken together, the above results illustrate that HOTAIR directly targets miR-217 to regulate its expression.

miR-217 was involved in the regulation of HOTAIR on cell proliferation and cycle arrest in HepG2 cells. To investigate whether the effects of HOTAIR on cell proliferation and cycle arrest in HepG2 cells were mediated by miR-217, we 
nCRNA HOTAIR 5'-aaTAATGTATTTTC - CATGCAGTt-3'

$:|||:|||:|\quad|||||||$

miRNA miR-217 3'-agGTTA-GTCAAGGACTACGTCAt-5

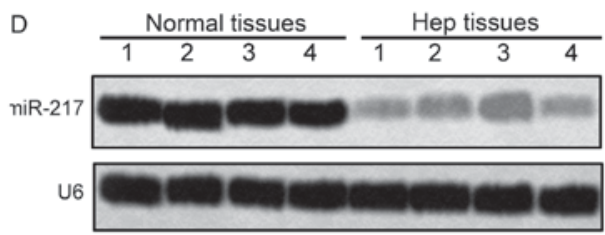

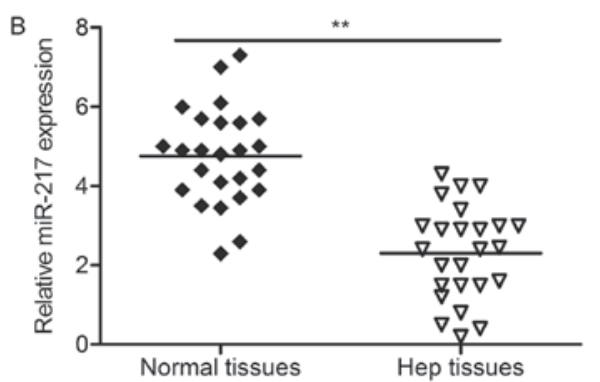
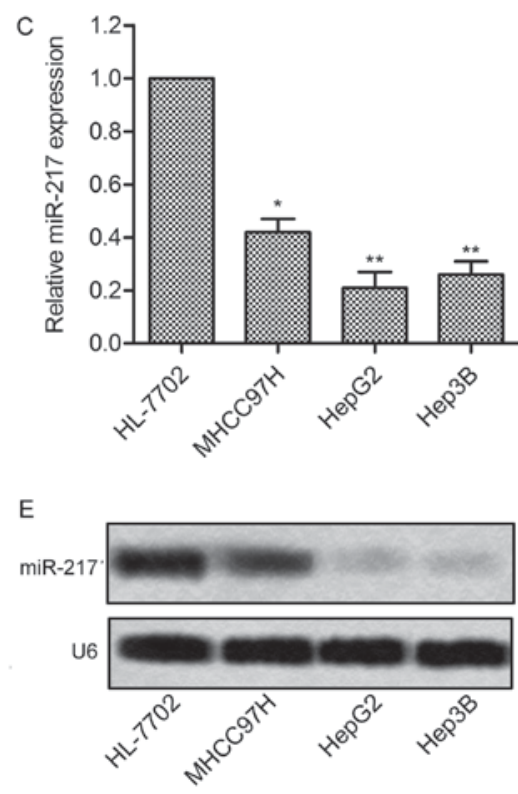

Figure 4. Expression of miR-217 was downregulated in liver cancer tissues and liver cancer cell lines. (A) The targeting sequences of HOTAIR with has-miR-217 were analyzed by StarBase v2.0 (http://starbase.sysu.edu.cn/). (B and C) The expression of miR-217 was evaluated by qRT-RCR in tissues and cell lines, respectively. (D and E) The expression of miR-217 was evaluated by northern blottimg in tissues and cell lines, respectively ("P<0.05, $\left.{ }^{* *} \mathrm{P}<0.01\right)$. HOTAIR, HOX transcript antisense RNA. miR-217, microRNA-217.
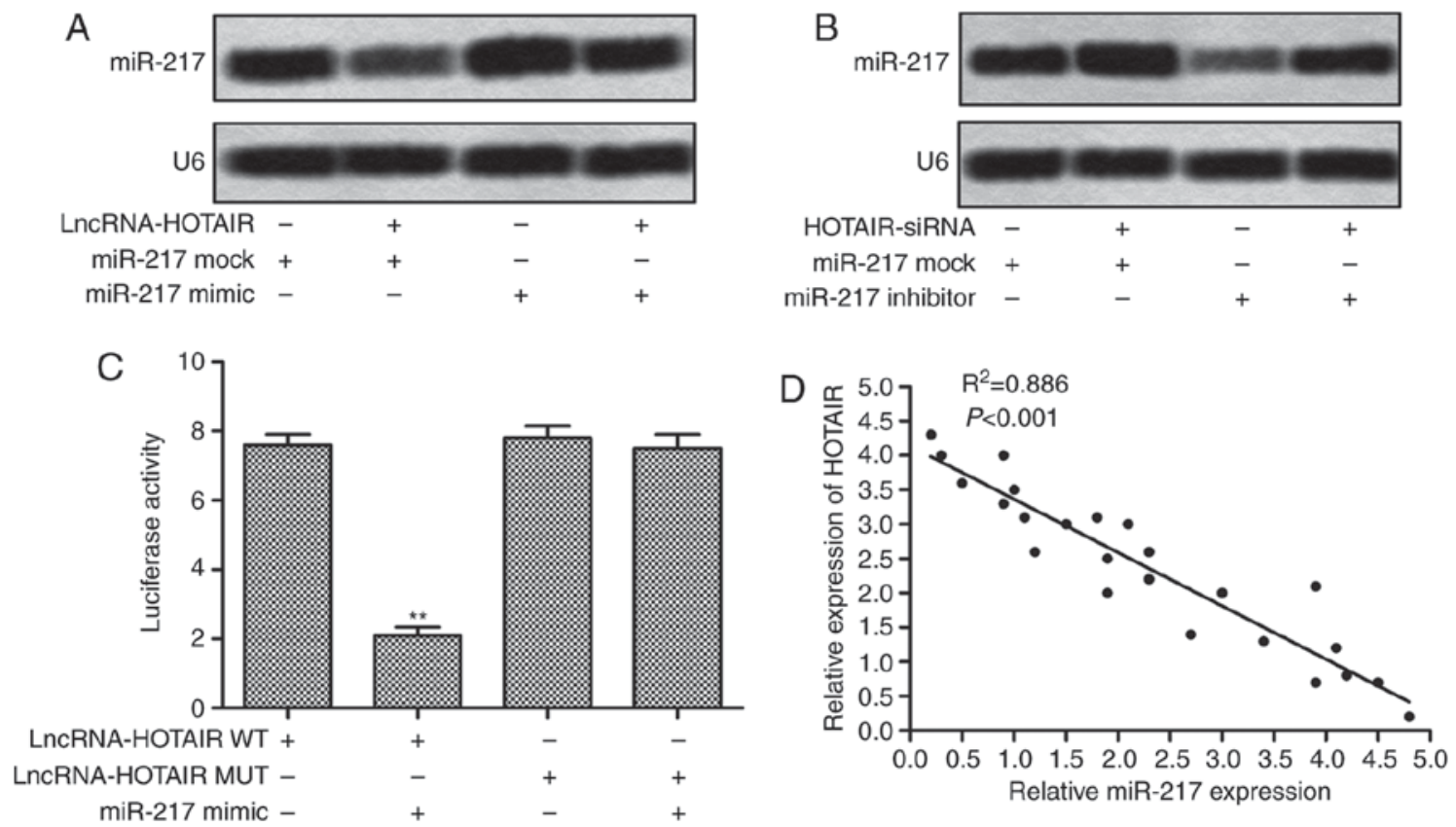

Figure 5. HOTAIR directly targeted miR-217. (A) HepG2 cells were transfected with miR-217 mimics or miR-217 mock alone, or co-transfected with HOTAIR siRNA lentiviral vectors together. The expression of miR-217 was evaluated by northern blotting. (B) HepG2 cells were transfected with miR-217 inhibitors or miR-217 mock alone, or co-transfected with HOTAIR siRNA lentiviral vectors together. The expression of miR-217 was evaluated by northern blotting. (C) HepG2 cells were co-transfected with pGL3 plasmids containing HOTAIR (WT or MUT) sequences and miR-217 mimics. The luciferase activity was detected by luciferase reporter assay. (D) The expression correlation between HOTAIR and mir-217 was determined in 25 liver cancer tissues ( ${ }^{* *} \mathrm{P}<0.01$ ). HOTAIR, HOX transcript antisense RNA. miR-217, microRNA-217.

transfected HepG2 cells with HOTAIR siRNA lentiviral vectors, miR-217 inhibitors, or the combination of lentiviral vectors and inhibitors. CCK- 8 assay and cell counting showed that inhibition of HOTAIR suppressed the proliferation 
A

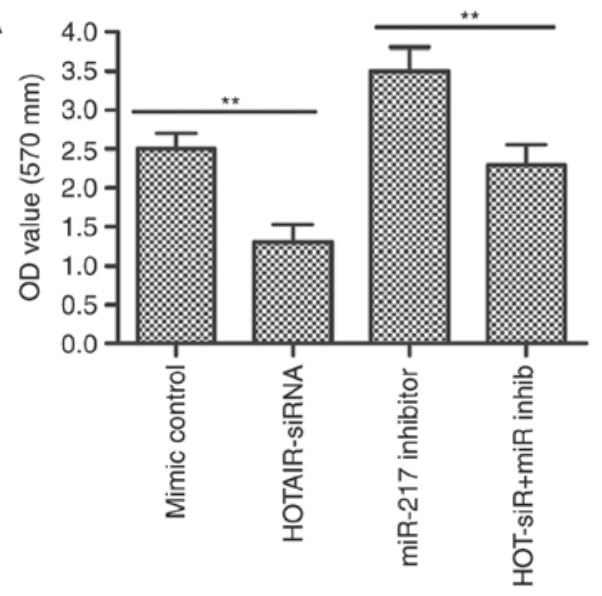

C
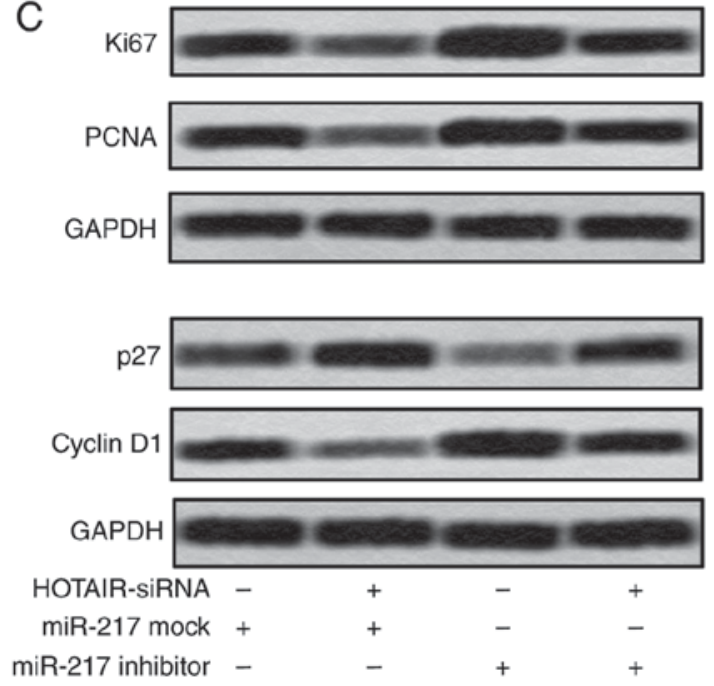

B

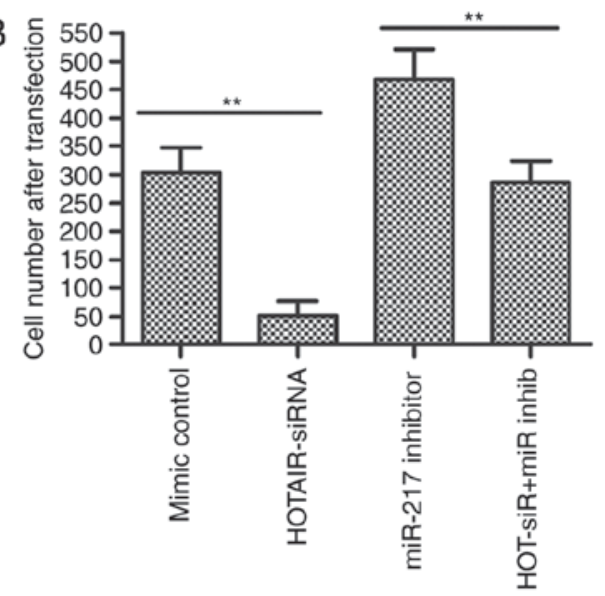

D

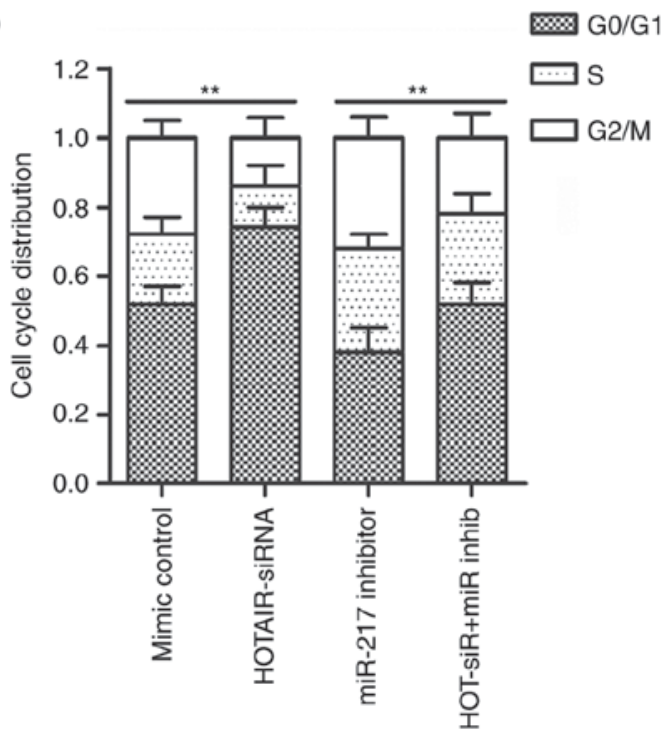

Figure 6. miR-217 was involved in the regulation of HOTAIR on cell proliferation and cycle arrest in HepG2 cells. HepG2 cells were transfected with HOTAIR siRNA lentiviral vectors or miR-217 inhibitors, or the combination. (A) The proliferation rate was measured by CCK-8 assay. (B) The cell number was assessed by optical microscope. (C) The protein expression of Ki67, PCNA, p27 and cyclin D1 was evaluated by western blotting. (D) The cell cycle arrest was evaluated by flow cytometry ( $(* \mathrm{P}<0.01)$. HOTAIR, HOX transcript antisense RNA. miR-217, microRNA-217.

and reduced cell numbers of HepG2 cells, while inhibition of miR-217 promoted the proliferation and increased cell numbers compared with control group. However, the proliferation and cell numbers in HOTAIR siRNA plus miR-217 inhibitor group and control group showed no significantly differences (Fig. 6A and B). As shown in Fig. 6C, western blotting results revealed that the protein expression levels of Ki67 and PCNA were downregulated in HOTAIR siRNA group and upregulated in miR-217 inhibitor group, while the levels did not significantly altered in HOTAIR siRNA plus miR-217 inhibitor group as compared with control group. Furthermore, the expression of p27 showed an obvious downregulation and the level of cyclin D1 showed an obvious upregulation in miR-217 inhibitor group, which were significantly reversed when co-transfection with HOTAIR siRNA lentiviral vectors and miR-217 inhibitors. Moreover, miR-217 inhibition-induced reduction of G0/G1 cycle arrest was partly rescued by inhibition of HOTAIR (Fig. 6D). Taken together, these observations suggest that the effects of HOTAIR on cell proliferation and cycle arrest in HepG2 cells were mediated by miR-217.
HOTAIR inhibition suppresses tumor growth in vivo. To further determine whether inhibition of HOTAIR could suppress the progression of liver cancer, an in vivo tumor model injected with HepG2 cells or HOTAIR-siRNA cells was used. Tumor volumes were measured every 6 days until the 30th day following cells injection. Five representative tumors from each group at 30th day were photographed and shown in Fig. 7A. The tumors volumes in HOTAIR-siRNA group were significantly smaller than that in control group (Fig. 7B). We then measured the expression of HOTAIR and miR-217 in the tumor tissues, and the results showed that the expression of HOTAIR was markedly reduced, while the expression of miR-217 was significantly increased in HOTAIR-siRNA tumor tissues as compared with control tumor tissues (Fig. 7C-F). In addition, we further performed immunohistochemistry assay to check the expression of Ki67 and PCNA in the tumor tissues. As shown in Fig. 7G and H, compared with control tumor tissues, Ki67 and PCNA positive cells had an obvious reduction in HOTAIR-siRNA tumor tissues. These in vivo results together with in vitro results suggest that inhibition of 

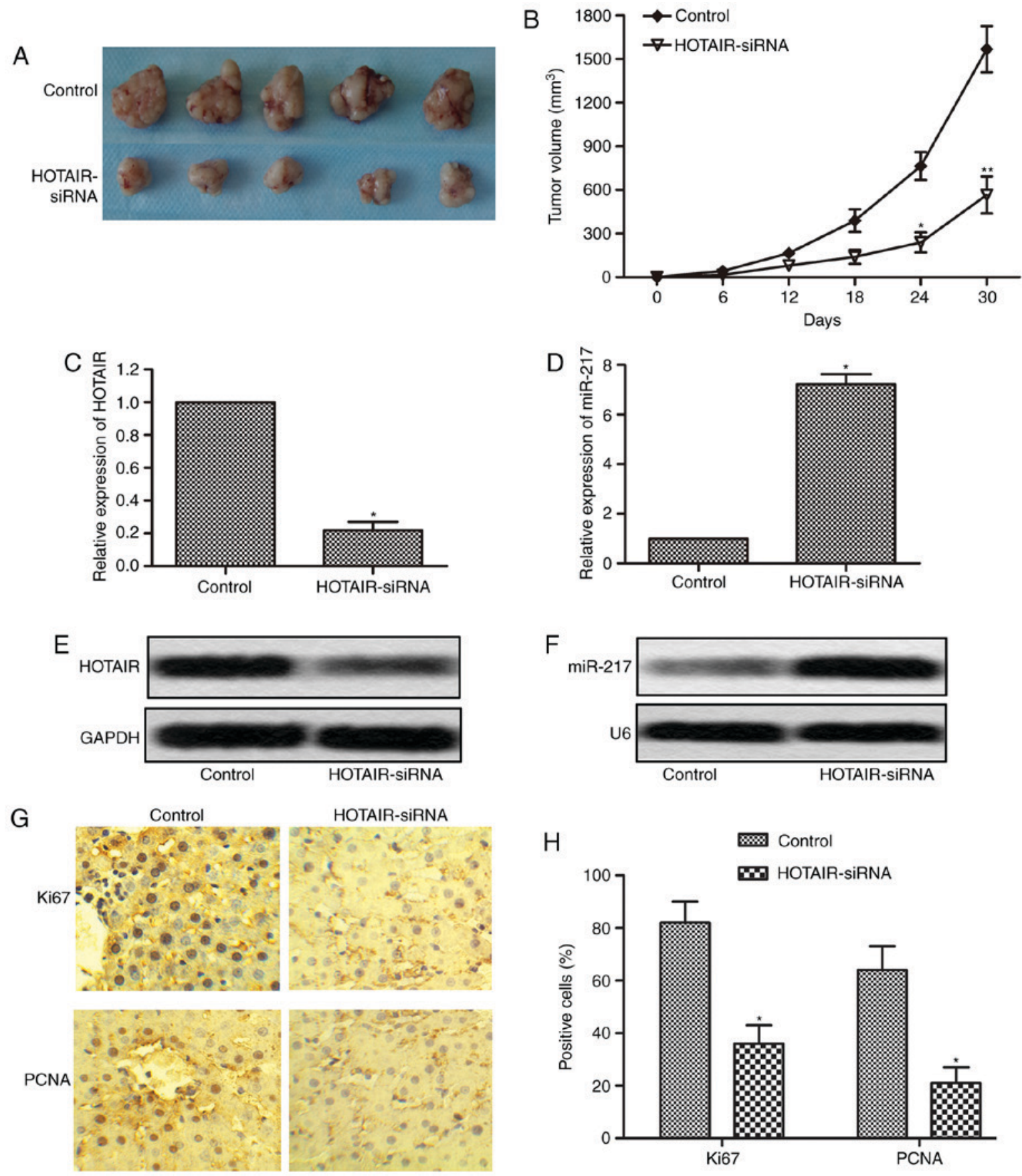

Figure 7. HOTAIR inhibition suppresses tumor growth in xenograft models. Xenograft mouse model was created by subcutaneous injection of HepG2 cells pretreated with or without HOTAIR siRNA lentiviral vectors to nude mouse. (A) Five primary tumors from each group were photographed after sacrifice at 30th day. (B) Tumor growth curves in two groups were shown every 6 days until the 30th day following cells injection. (C and D) The expression of HPTAIR and miR-217 in primary tumor tissues was measured by qRT-PCR. (E and F) The expression of HPTAIR and miR-217 in primary tumor tissues was measured by northern blotting. ( $\mathrm{G}$ and $\mathrm{H}$ ) The expression of Ki67 and PCNA in primary tumor tissues was visualized by immunohistochemistry $\left({ }^{*} \mathrm{P}<0.05,{ }^{* *} \mathrm{P}<0.01\right)$. HOTAIR, HOX transcript antisense RNA. miR-217, microRNA-217.

HOTAIR suppresses tumor growth by regulating the expression of miR-217.

\section{Discussion}

In recent years, a variety of investigations have reported that lncRNAs have significant functions in cancer pathogenesis. HOTAIR functions as an oncogenic lncRNA and it has been studied in many cancers including liver cancer. However, its biological roles and underlying molecular mechanisms in liver cancer are still largely unknown. Previous studies have shown that the expression of HOTAIR was upregulated in many cancer types including liver cancer. In the present study, we found that the expression of HOTAIR had an significantly upregulation in the liver cancer tissues and liver cancer cell lines, MHCC 97H, HepG2 and Hep3B, which was consistent with the previous results. These data confirm that high level of HOTAIR is closely associated with the progression of liver cancer, suggesting that HOTAIR inhibition may be an effective therapeutic strategy for liver cancer. 
Previous reports have demonstrated that HOTAIR plays an important role in cancers by regulating cellular events, such as cell proliferation, cell invasion and tumor metastasis. HOTAIR promotes human liver cancer stem cell malignant growth through downregulation of SETD2 (26). HOTAIR inhibition in liver cancer cells leads to the suppression of cell proliferation and invasion in vitro, and significantly inhibited the rate of growth of liver cancer cells in vivo, partly through the regulation of the $\mathrm{Wnt} / \beta$-catenin signaling pathway (27). HOTAIR could promote migration and invasion of liver cancer cells by inhibiting RBM38. These studies suggest that HOTAIR regulates the development and progression of liver cancer through many different signal molecules or pathways. In the present study, to further explore the biological effects of HOTAIR on the proliferation and cycle arrest in liver cancer, HOTAIR siRNA lentiviral vectors were used to infect HepG2 cells to interfere the expression of HOTAIR. CCK- 8 assay and cell counting revealed that the cell proliferation had an obvious suppression, and the cell number had an obvious reduction in HOTAIR-siRNA group as compared with control group. Western blot assay showed that compared with the control group, the protein expression levels of Ki67 and PCAN, two proliferation-associated markers, were significantly decreased in HOTAIR-siRNA group. Moreover, flow cytometry assay showed that the G0/G1 cell cycle arrest was significantly increased in HOTAIR-siRNA group. The protein expression level of p27 had an obvious upregulation, while the level of cyclin D1 had a significant downregulation in HOTAIR-siRNA group as compared with control group. These results suggest that HOTAIR inhibition suppresses cell proliferation and induces G0/G1 cell cycle arrest in HepG2 cells.

LncRNAs have been reported to exert various biological functions by targeting miRNAs. HOTAIR participates in inhibiting the expression of miR-205, which has a negative correlation with the degree of bladder cancer malignancy by regulating proliferation, migration and invasion of bladder cancer cells (28). HOTAIR promotes the progression of glioma by inhibiting miR-326, and further upregulated the expression of firoblast growth factor 1 (FGF1) (29). Also, the oncogenic activity of HOTAIR in gallbladder cancer is partly through repressing miRNA-130a (30). These reports demonstrate that the biological activities of HOTAIR in different cancer types can be mediated miRNAs. Recently, miR-217 was shown to be involved in the development of liver cancer. Moreover, miR-217 has been reported to be downregulated in many cancers, such as lung cancer, colorectal cancer, epithelial ovarian cancer, and pancreatic cancer and function as a tumor suppressor gene (31-34). In this study, we explored the interaction between HOTAIR and miR-217 by bioinformatics prediction and experiments, and found that HOTAIR directly targeted miR-217 to regulate its expression. The expression of miR-217 had an significantly downregulation in the liver cancer tissues and liver cancer cell lines, and the expression levels of HOTAIR and miR-217 were inversely correlated in liver cancer tissues, which further supported the regulation of miR-217 by HOTAIR. We further investigated whether the effects of HOTAIR on cell proliferation and cycle arrest in HepG2 cells were mediated by miR-217. The results showed that the effects of HOTAIR inhibition on proliferation suppression and G0/G1 cycle arrest induction in HepG2 cells were in part rescued by miR-217 inhibition. Taken together, these observations suggest that the effects of HOTAIR on cell proliferation and cycle arrest in HepG2 cells were mediated by $\mathrm{miR}-217$.

To further determine whether inhibition of HOTAIR could suppress the progression of liver cancer, an in vivo tumor model was used. And in vivo experiments had shown that HOTAIR inhibition significantly decreased the tumors volumes, and the expression of miR-217 was increased, the expression of Ki67 and PCNA was reduced in HOTAIR-siRNA tumor tissues. These in vivo results are consistent with the data in vitro experiments, and suggest that inhibition of HOTAIR suppresses liver cancer progression and growth by regulating the expression of miR-217.

In conclusion, the present study demonstrates that HOTAIR expression is upregulated in liver cancer tissues, and functions as an oncogene in liver cancer. HOTAIR inhibition significantly suppresses the progression of liver cancer by inhibiting cell proliferation and inducing G0/G1 cell cycle arrest of liver cancer cells, which was partly mediated by upregulating miR-217. Thus, targeting HOTAIR-miR-217 axis may provide novel insight into therapeutic targets for the treatment of liver cancer.

\section{Acknowledgements}

The authors thank the Xi'an No. 4 Hospital for providing technical support.

\section{Funding}

No funding was received.

\section{Availability of data and materials}

All data generated or analyzed during this study are included in this published article.

\section{Authors' contributions}

LW and JW performed the examinations and analyzed the data. XW contributed to analysis of the data, and was a major contributor in writing the manuscript. All authors read and approved the final manuscript.

\section{Ethics approval and consent to participate}

Informed consent was obtained from all the patients. The study was performed in accordance with the Helsinki Declaration and was approved by the Human Ethics Committee/Institutional Review Board of Xi'an Honghui Hospital.

\section{Consent for publication}

Informed consent was obtained from all the patients.

\section{Competing interests}

The authors declare that they have no competing interests. 


\section{References}

1. Bosch FX, Ribes J, Díaz M and Cléries R: Primary liver cancer: Worldwide incidence and trends. Gastroenterology 127: S5-S16, 2004.

2. Dong C, Zhao B, Long F, Liu Y, Liu Z, Li S, Yang X, Sun D, Wang $\mathrm{H}$, Liu $\mathrm{Q}$, et al: Nogo-B receptor promotes the chemoresistance of human liver cancer via the ubiquitination of $\mathrm{p} 53$ protein Oncotarget 7: 8850-8865, 2016.

3. Zheng Z, Liu J, Yang Z, Wu L, Xie H, Jiang C, Lin B, Chen T, Xing C, Liu Z, et al: MicroRNA-452 promotes stem-like cells of liver cancer by inhibiting Sox7 involving Wnt/ $\beta$-catenin signaling pathway. Oncotarget 7: 28000-28012, 2016.

4. Qin Y, Zhao D, Zhou HG, Wang XH, Zhong WL, Chen S, Gu WG, Wang W, Zhang CH, Liu YR, et al: Apigenin inhibits $\mathrm{NF}-\kappa \mathrm{B}$ and snail signaling, EMT and metastasis in human liver cancer. Oncotarget 7: 41421-41431, 2016.

5. Yang Z, Zhou L, Wu LM, Lai MC, Xie HY, Zhang F and Zheng SS: Overexpression of long non-coding RNA HOTAIR predicts tumor recurrence in liver cancer patients following liver transplantation. Ann Surg Oncol 18: 1243-1250, 2011.

6. Mercer TR, Dinger ME and Mattick JS: Long non-coding RNAs: Insights into functions. Nat Rev Genet 10: 155-159, 2009.

7. Gibb EA, Brown CJ and Lam WL: The functional role of long non-coding RNA in human carcinomas. Mol Cancer 10: 38, 2011.

8. Zhang TH, Liang LZ, Liu XL, Wu JN, Su K, Chen JY, Zheng QY, Huang HZ and Liao GQ: Long non-coding RNA MALAT1 interacts with miR-124 and modulates tongue cancer growth by targeting JAG1. Oncol Rep 37: 2087-2094, 2017.

9. Li Y, Wu Z, Yuan J, Sun L, Lin L, Huang N, Bin J, Liao Y and Liao W: Long non-coding RNA MALAT1 promotes gastric cancer tumorigenicity and metastasis by regulating vasculogenic mimicry and angiogenesis. Cancer Lett 395: 31-44, 2017.

10. Jin Y, Cui Z, Li X, Jin X and Peng J: Upregulation of long non-coding RNA PlncRNA-1 promotes proliferation and induces epithelial-mesenchymal transition in prostate cancer. Oncotarget 8: 26090-26099, 2017.

11. Wei W, Liu Y,Lu Y, Yang B and Tang L: LncRNA XIST promotes pancreatic cancer proliferation through miR-133a/EGFR. J Cell Biochem 118: 3349-3358, 2017.

12. Xue X, Yang YA, Zhang A, Fong KW, Kim J, Song B, Li S, Zhao JC and Yu J: LncRNA HOTAIR enhances ER signaling and confers tamoxifen resistance in breast cancer. Oncogene 35 2746-2755, 2016

13. Kogo R, Shimamura T, Mimori K, Kawahara K, Imoto S, Sudo T, Tanaka F, Shibata K, Suzuki A, Komune S, et al: Long noncoding RNA HOTAIR regulates polycomb-dependent chromatin modification and is associated with poor prognosis in colorectal cancers. Cancer Res 71: 6320-6326, 2011.

14. Kim K, Jutooru I, Chadalapaka G, Johnson G, Frank J, Burghardt R, Kim S and Safe S: HOTAIR is a negative prognostic factor and exhibits pro-oncogenic activity in pancreatic cancer. Oncogene 32: 1616-1625, 2013.

15. Liu X, Liu Z, Sun M, Liu J, Wang Z and Wei D: The long non-coding RNA HOTAIR indicates a poor prognosis and promotes metastasis in non-small cell lung cancer. BMC Cancer 13: 464, 2013

16. Chen FJ, Sun M, Li SQ, Wu QQ, Ji L, Liu ZL, Zhou GZ, Cao G, Jin L, Xie HW, et al: Upregulation of the long non-coding RNA HOTAIR promotes esophageal squamous cell carcinoma metastasis and poor prognosis. Mol Carcinog 52: 908-915, 2013.

17. Gao J, Jia LI, Jingli DU and Xiaolei LI: Long non-coding RNA HOTAIR is a marker for liver cancer progression and tumor recurrence. Oncol Lett 11: 1791-1798, 2016.
18. Geng YJ, Xie SL, Li Q, Ma J and Wang GY: Large Intervening non-coding RNA HOTAIR is associated with liver cancer progression. J Int Med Res 39: 2119-2128, 2011.

19. Bartel DP: MicroRNA target recognition and regulatory functions. Cell 136: 215-233, 2009.

20. Calin GA and Croce CM: MicroRNA signatures in human cancers. Nat Rev Cancer 6: 857-866, 2006.

21. Su DN, Wu SP, Chen HT and He JH: HOTAIR, a long non-coding RNA driver of malignancy whose expression is activated by FOXC1, negatively regulates miRNA-1 in liver cancer. Oncol Lett 12: 4061-4067, 2016.

22. Su J, Wang Q, Liu Y and Zhong M: miR-217 inhibits invasion of liver cancer cells through direct suppression of E2F3. Mol Cell Biochem 392: 289-296, 2014

23. López-Terrada D, Cheung SW, Finegold MJ and Knowles BB: HepG2 is a hepatoblastoma-derived cell line. Hum Pathol 40: 1512-1515, 2009.

24. Zhou Y, Fukuda T, Hang Q, Hou S, Isaji T, Kameyama A and $\mathrm{Gu} \mathrm{J}$ : Inhibition of fucosylation by 2-fluorofucose suppresses human liver cancer HepG2 cell proliferation and migration as well as tumor formation. Sci Rep 7: 11563, 2017.

25. Shao LW, Huang LH, Yan S, Jin JD and Ren SY: Cordycepin induces apoptosis in human liver cancer HepG2 cells through extrinsic and intrinsic signaling pathways. Oncol Lett 12: 995-1000, 2016.

26. Li H, An J, Wu M, Zheng Q, Gui X, Li T, Pu H and Lu D: LncRNA HOTAIR promotes human liver cancer stem cell malignant growth through downregulation of SETD2. Oncotarget 6: 27847-27864, 2015.

27. Gao JZ, Li J, Jl DU and Li XL: Long non-coding RNA HOTAIR is a marker for liver cancer progression and tumor recurrence. Oncol Lett 11: 1791-1798, 2016.

28. Sun X, Du P, Yuan W, Du Z, Yu M, Yu X and Hu T: Long non-coding RNA HOTAIR regulates cyclin J via inhibition of microRNA-205 expression in bladder cancer. Cell Death Dis 6: e1907, 2015.

29. Ke J, Yao Y, Zheng J, Wang P, Liu YH, Ma J, Li Z, Liu XB, Li ZQ, Wang ZH and Xue YX: Knockdown of long non-coding RNA HOTAIR inhibits malignant biological behaviors of human glioma cells via modulation of miR-326. Oncotarget 6 : 21934-21949, 2015.

30. Ma MZ, Li CX, Zhang Y, Weng MZ, Zhang MD, Qin YY, Gong W and Quan ZW: Long non-coding RNA HOTAIR, a c-Myc activated driver of malignancy, negatively regulates miRNA-130a in gallbladder cancer. Mol Cancer 13: 156, 2014.

31. Guo J, Feng Z, Huang Z, Wang H and Lu W: MicroRNA-217 functions as a tumour suppressor gene and correlates with cell resistance to cisplatin in lung cancer. Mol Cells 37: 664-671, 2014.

32. Wang B, Shen ZL, Jiang KW, Zhao G, Wang CY, Yan YC, Yang Y, Zhang JZ, Shen C, Gao ZD, et al: MicroRNA-217 functions as a prognosis predictor and inhibits colorectal cancer cell proliferation and invasion via an AEG-1 dependent mechanism. BMC Cancer 15: 437, 2015.

33. Li J, Li D and Zhang W: Tumor suppressor role of miR-217 in human epithelial ovarian cancer by targeting IGF1R. Oncol Rep 35: 1671-1679, 2016.

34. Deng S, Zhu S, Wang B, Li X, Liu Y, Qin Q, Gong Q, Niu Y, Xiang C, Chen J, et al: Chronic pancreatitis and pancreatic cancer demonstrate active epithelial-mesenchymal transition profile, regulated by miR-217-SIRT1 pathway. Cancer Lett 355: 184-191, 2014. 